\title{
Estudio fitoquímico de hojas de Uncaria guianensis y evaluación de actividad antibacteriana
}

\author{
Juliet Angélica PRIETO RODRÍGUEZ', Oscar Javier PATIÑO LADINO², Liliana LESMES³, \\ Jose Manuel LOZANO ${ }^{4}$, Luis Enrique CUCA SUÁREZ ${ }^{5}$
}

\section{RESUMEN}

Del extracto de éter de petróleo de hojas de Uncaria guianensis (Rubiaceae), se aisló un compuesto tipo clorina denominado éster etílico de feoforbida $a$ y una mezcla de esteroles conocidos como $\beta$-sitosterol y estigmasterol. Sus estructuras fueron elucidadas por análisis detallado de RMN, incluyendo técnicas bidimensionales, y por comparación con datos reportados en la literatura. Posteriormente, se evaluó la actividad antibacteriana al éster etílico de feoforbida a contra dos cepas Gram (+): $S$. aureus ATCC 6538 y E. faecalis ATCC 29212 y contra tres cepas Gram (-): E. coli ATCC 25922, S. typhimurium ATCC 14028s y S. typhimurium MS7953. Se encontró actividad significativa contra S. aureus, E. faecalis, E. coli y S. tiphymurium MS7953. PALABRAS-CLAVE: Uncaria guianensis, Rubiaceae, clorina, éster etílico de feoforbida a, actividad antibacteriana.

\section{Phytochemical study of Uncaria guianensis leaves and antibacterial activity evaluation}

\begin{abstract}
A chlorin compound, pheophorbide $a$ ethyl ester and a mixture of sterols known as $\beta$-sitosterol and stigmasterol, were isolated from the petroleum ether extract of Uncaria guianensis (Rubiaceae) leaves. Their structures were elucidated by detailed analysis of NMR spectra, including bidimensional techniques and by comparison with literature data. The antibacterial activity for the pheophorbide $a$ ethyl ester was evaluated against two Gram (+) strains: S. aureus ATCC 6538 y E. faecalis ATCC 29212 and three Gram (-) strains: E. coli ATCC 25922, S. typhimurium ATCC 14028s y S. typhimurium MS7953S. aureus ATCC 6538 and E. fecalis ATCC 29212, finding significant activity against S. aureus 6538, E. faecalis 29212, S. tiphymurium MS7953 and E. coli 25922.
\end{abstract}

KEYWORDS: Uncaria guianensis, Rubiaceae, chlorin, pheophorbide a ethyl ester, antimicrobial activity.

\footnotetext{
1 Universidad Nacional de Colombia. japrietor@unal.edu.co

2 Universidad Nacional de Colombia. ojpatinol@unal.edu.co

3 FIDIC. liliana_lesmes_s@fidic.org.co

${ }^{4}$ Universidd Nacional de Colombia. jmlozanom@unal.edu.co

5 Universidad Nacional de Colombia. lecucas@unal.edu.co
} 


\section{INTRODUCCIÓN}

El género Uncaria, perteneciente a la familia Rubiaceae, comprende cerca de 60 especies distribuidas principalmente en África y Asia. Uncaria guianensis y U. tomentosa, están presentes en amplias zonas de la Amazonía brasilera y en otros países americanos como: Guatemala, Honduras, El Salvador, Nicaragua, Costa Rica, Panamá Colombia, Venezuela, Ecuador, Perú y Bolivia (Zeballos y Tomazello 2006). El término Uncaria proviene del latín uncus que significa gancho, en alusión a las espinas a lo largo del tallo que poseen las especies de este género (Rojas 2001). En Colombia se encuentran reportadas 2 especies de este género, $U$. guianensis y $U$. tomentosa, que crecen en bosques húmedos de la Amazonía, Chocó, Antioquia, Caquetá, Cundinamarca, Bolívar, Santander y Magdalena medio (Mendoza et al. 2004). Estas especies son consideradas potencial económico de la Amazonía, debido a que todas las partes de la planta (hojas, corteza y raíz) son aprovechables y a que son empleadas ampliamente en medicina tradicional, especialmente en Sur América (Rojas 2001). Diferentes especies del género Uncaria son ampliamente utilizadas en medicina tradicional para tratar diversas dolencias (Aquino et al. 1991; Laus et al. 1997; Keplinger et al. 1999; Heitzman et al. 2005; Trejo et al. 2005; Pilarski et al. 2006), entre las cuales se destaca la especie $U$. tormentosa, a partir de la cual se han elaborado diferentes fitomedicamentos que es posible encontrar en el mercado, estos medicamentos se emplean principalmente para el tratamiento de enfermedades cardiovasculares (Wang 1989; Huang 1999), ginecológicas, renales, gastrointestinales, hepáticas y reumáticas (Keplinger et al. 1999), además como estimulantes inmunitarios, antimicrobianos y antivirales (Aquino et al. 1989). De los estudios fitoquímicos realizados en algunas especies del género Uncaria, se han encontrado principalmente alcaloides oxindólicos tetracíclicos y pentacíclicos (Heitzman et al. 2005; Muhammad et al. 2001; Aquino et al. 1991), alcaloides indólicos (Aquino et al. 1991) y $\beta$-carbonilícos (Pengsuparp et al. 2001), flavonoides (Heitzman et al. 2005), cumarinas (Valente et al. 2009), protoantocianidinas (Gonçalves et al. 2005), taninos (Pilarski et al. 2006), esteroides (Senatore et al. 1989), triterpenoides derivados del ursano (Heitzman et al. 2005; Álvarez et al. 1988) y glicósidos de ácido quinovico. Para los extractos y compuestos aislados de algunas especies de este género, se ha reportado que presentan actividad citotóxica, antiinflamatoria, antiviral, antibacteriana, hipotensiva, antioxidante, inmunoestimulante, anticonvulsiva y neuroprotectora (Aquino et al. 1991; Laus et al. 1997; Keplinger et al. 1999; Heitzman et al. 2005; Trejo et al. 2005; Shim et al. 2009; Dreifuss et al. 2010).

$U$. guianensis es una de las especies del género Uncaria presente en el territorio colombiano. Esta especie también se conoce con el nombre de uńa de gato, garabato, bejuco de agua, jala pa tras y unganangui (Mendoza et al. 2004; Rojas 2001). Los indígenas peruanos, específicamente la tribu Ashaninka, emplean el extracto acuoso de la corteza de $U$. guianensis para el tratamiento de cáncer, diabetes, artritis e inflamaciones (Heitzman et al. 2005; Rojas 2001). Para esta especie se reportan como metabolitos secundarios principalmente alcaloides oxindólicos (Lavault et al. 1983; Laus y Keplinger 2003; Carbonezi et al. 2004; Heitzman et al. 2005; Pereira et al. 2008), triterpenos petacíclicos (Heitzman et al. 2005), flavonoides (Álvarez et al. 1988; Heitzman et al. 2005; Valente et al. 2009) y glicósidos de ácido quinóvico (Yépez et al. 1991; Heitzman et al. 2005). En un estudio realizado por Valente y colaboradores en 2009, se determinó que el flavonoide glicosidado kaempferitrina solo es producido por U. guianensis, lo que indica que esta sustancia y posiblemente otros flavonoides glicósidados pueden ser de valor quimiotaxonómico para la especie.

En este trabajo se reporta el aislamiento y caracterización de un compuesto tipo clorina denominado éster etílico de feoforbida $a$, junto con dos esteroles conocidos como $\beta$-sitosterol y estigmasterol, los cuales se reportan por primera vez para la especie. También se reporta la evaluación de actividad antibacteriana del éster etílico de feoforbida $a$.

\section{MATERIAL Y MÉTODOS}

\section{Métodos químicos y físicos}

Los espectros RMN de ${ }^{1} \mathrm{H},{ }^{13} \mathrm{C}$, junto con los espectros bidimensionales (COSY, DEPT, HMQC y HMBC), fueron tomados en el equipo Bruker Avance 400, a $400 \mathrm{MHz}$ para ${ }^{1} \mathrm{H}$ y a $100 \mathrm{MHz}$ para ${ }^{13} \mathrm{C}$. El espectro visible fue tomado en un equipo Lambda 2S. Los espectros IR fueron tomados en película en el equipo Perkin Elmer FTIR Panagon 500. Los espectros de masas de alta resolución fueron tomados en un espectrómetro de masas acoplado a un cromatógrafo líquido LC-MS-IT-TOF Shimadzu por inyección directa usando ionización por ESI en modo positivo. Los puntos de fusión se tomaron en el fusiómetro Mel-TEmp. Para cromatografía en columna se empleó sílica gel (Kieselgel 60 Merck) y para cromatografía en capa delgada sílica gel (Kieselgel 60 GF254 Merck) de 0,3 mm de espesor, para el caso de CCDP se empleó $1 \mathrm{~mm}$ espesor. Se empleó luz UV ( $\lambda 254$ y $365 \mathrm{~nm}$ ) y vapores de yodo como reveladores universales.

Para la obtención de cromatogramas de gases se empleó el cromatógrafo 6C-FID-FPD Shimadzu, con las siguientes condiciones cromatográficas: Columna: RTX-S $15 \mathrm{~m}$ x 0,25 mm di; Gas de arrastre: Helio; Detector: FID; T. inyector y detector: $300^{\circ} \mathrm{C}$; T. columna: $135-300^{\circ} \mathrm{C}\left(4^{\circ} \mathrm{C} \mathrm{min}^{-1}\right)$.

\section{Material vegetal}

Las hojas de Uncaria guianensis (Rubiaceae) fueron recolectadas en el campus de la Universidad Nacional de 
Colombia - Sede Leticia (Amazonía Colombiana), por la Bióloga Adriana Aguilar en Junio de 2006. Fue determinada por el Botánico Adolfo Jara en Octubre de 2006. Un espécimen reposa en el Herbario Nacional Colombiano del Instituto de Ciencias Naturales de la Universidad Nacional Colombiano con el número de colección COL-518190.

\section{Extracción y aislamiento}

Las hojas secas y molidas $(2 \mathrm{Kg})$ fueron sometidas a extracción por maceración con etanol al 96\%. El extracto etanólico resultante fue concentrado a presión reducida, obteniendo $192 \mathrm{~g}$ de extracto crudo. $60 \mathrm{~g}$ de extracto crudo se sometió a fraccionamiento por extracción sólido-líquido tipo Soxhlet con solventes de polaridad creciente, éter de petróleo (EdP), cloroformo $\left(\mathrm{CHCl}_{3}\right)$, acetato de isopropilo (AcOiPr) y metanol $(\mathrm{MeOH})$. La fracción de $\operatorname{EdP}(4,8 \mathrm{~g})$ fue sometida a cromatrografía en columna eluida con toluenoAcOiPr (9:1) incrementando la polaridad hasta toluenoAcOiPr (1:1), obteniendo 14 fracciones, denominadas E1 a E14. Las fracciones E5 y E6 fueron reunidas $(900 \mathrm{mg})$ y sometidas a cromatografía en columna (tolueno-AcOiPr en polaridad creciente 9:1 a 6:4) para obtener 29 fracciones, denominadas E5-1 a E5-29. La fracción E5-14 se lavó con acetona para obtener un sólido blanco cristalino $(30 \mathrm{mg})$, con punto de fusión $138-141^{\circ} \mathrm{C}$, sólido que corresponde a la mezcla de $\beta$-sitosterol $(82,13 \%)$ y estigmasterol $(17,87 \%)$ (De Andrade 2003).

La fracción E7 de (180 mg) fue sometida a cromatografía en columna repetitiva en sílica gel (hexano-acetona 7:3; EdP-AcOiPr 6:4), cromatografía en columna con Sephadex LH-20 eluída con metanol y cromatografía en capa delgada preparativa (benceno-AcOiPr 8:2) para obtener $5 \mathrm{mg}$ del éster etílico de feoforbida a 1 (Spangler et al. 1977; Stermitz et al. 2000; Musumeci et al. 2003; Smith et al. 1987).

\section{Métodos Biológicos}

Cepas bacterianas. Gram (+): Staphylococcus aureus ATCC 65380, Enterococcus faecalis ATCC 29212. Gram (-): Escherichia coli ATCC 25922 y las cepas de Salmonella typhimurium ATCC 14028s y sus mutantes MS 7953 y EG10627 aportadas para este estudio por el Profesor E. A Groisman del Departamento de Microbiología Molecular de la Universidad de Washington, St. Louis, MO, USA.

\section{Medios para cultivo bacteriano y otros reactivos}

Para los cultivos y subcultivos de las cepas Gram (+) se utilizó Caldo de Tripticasa de soya. (TSB; Difco Laboratories, Detroit, MI, USA). Para los cultivos y subcultivos de las cepas Gram (-) se empleó Base Luria Broth (LB; Gibco, Cerdanyola del valle, Barcelona, España).
Para realizar la dispersión bacteriana en los ensayos de difusión radial se empleó medio dispersivo bajo en nutrientes preparado con $10 \mathrm{~g}$ de agarosa de baja electroendoosmosis (Sigma Chem. Co, St. Louis, MO, USA), Tween-20 al 0,02\% (v/v) (Merck, Darmstadt, Alemania) y 0,3 g de TSB (Tripticasa de soya) (Difco Laboratories, Detroit, MI, USA) para un volumen final de un litro de solución.

Para la detección del crecimiento bacteriano se empleó un medio nutritivo revelador en una sobre capa mediante la mezcla de los siguientes componentes: $20 \mathrm{~g}$ de agar-agar (Difco Laboratories, Detroit, MI. USA), Tween-20 al 0,02\% (v/v) (Merck, Darmstadt, Alemania) y $10 \mathrm{~g}$ de Tripticasa de soya (TSB) (Difco Laboratories, Detroit, MI, USA). Buffer fosfato salino (PBS) $\mathrm{pH}$ 7,2- 7,4 se preparó de la siguiente manera: 0,2 g KH 2 PO4, $32 \mathrm{~g} \mathrm{NaCl}, 0,8 \mathrm{~g}$ de $\mathrm{KCl}, 4,6 \mathrm{~g}$ de $\mathrm{Na}_{2} \mathrm{HPO} 4$ para un volumen de un litro de solución.

Ampicilina 50,8 $\mathrm{mg} \mathrm{mL}^{-1}$, (Binotal; Bayer ${ }^{\circ}$ Alemania), Sulfato de Kanamicina 10,25 mg mL ${ }^{-1}$ (Gibco, Cerdanyola del valle, Barcelona, España), Tetraciclina monohidrato 4,12 $\mathrm{mg} \mathrm{mL}^{-1}$ (Sigma Chem. Co, St. Louis, MO, USA).

\section{Actividad Antibacteriana}

La actividad antibacteriana del éster etílico de feoforbida a se evaluó frente a dos cepas de bacterias Gram (+) y tres Gram (-).

La actividad antibacteriana del compuesto fue evaluada por la técnica de difusión radial adaptada de la metodología previamente publicada por Lehrer y colaboradores (Lehrer $e t$ al. 1991), frente a las cepas bacterianas descritas anteriormente. Se toma una colonia aislada de cada cepa y se siembra en 3 $\mathrm{mL}$ de tripticasa de soya (TSB) para las bacterias Gram (+) y 3 $\mathrm{mL}$ de LB para las bacterias $\operatorname{Gram}(-)$, se incuban a $37^{\circ} \mathrm{C}$ toda la noche con agitación, de este cultivo se toman $200 \mathrm{~mL}$ y se inoculan en $20 \mathrm{~mL}$ de TSB o LB según el caso, se incuban por 5 horas a $37^{\circ} \mathrm{C}$ con agitación hasta que los microorganismos se encuentren en la fase logarítmica, posteriormente se centrifugan a $10000 \mathrm{rpm}$ por 10 minutos a $4^{\circ} \mathrm{C}$, se retira el sobrenadante y el sedimento obtenido se resuspende en 10 $\mathrm{mL}$ de buffer de fosfato salino (PBS) $\mathrm{pH} 7,2$, seguidamente se realizan 3 lavados con PBS centrifugando a $10000 \mathrm{rpm}$ por 5 minutos cada uno. Finalmente el sedimento se resuspende en $5 \mathrm{~mL}$ de PBS pH 7,2 y se determinó la densidad óptica (DO) a $620 \mathrm{~nm}$, para calcular el número de UFC (Unidades formadoras de colonia) por mililitro. Basados en este valor se calculó el volumen que contiene 4 x $10^{7}$ UFC a ser dispersadas en cada plato teniendo en cuenta la relación: $\mathrm{DO}_{620 \mathrm{~nm}} 0.2=$ $5 \times 10^{7}$ UFC (Lehrer et al. 1991). El volumen medido se mezcló y homogenizó en $15 \mathrm{~mL}$ de agarosa de baja electroendoósmosis fundida a mas o menos $45^{\circ} \mathrm{C}$, esta suspensión bacteriana se sirvió en cajas de Petri y se dejó solidificar a temperatura ambiente, luego de lo cual se hicieron perforaciones de 2 
$\mathrm{mm}$ de diámetro con un sacabocados estéril. Para el ensayo las muestras se prepararon disolviendo $1 \mathrm{mg}$ del compuesto puro en $500 \mu \mathrm{L}$ de DMSO, de los cuales se colocan $8 \mu \mathrm{L}$ de la muestra por duplicado y se incuba a $37^{\circ} \mathrm{C}$ por 30 minutos. $\mathrm{Al}$ cabo de este tiempo se adiciona el medio nutritivo fundido el cual contiene agar-agar y TSB, se incuba por 18 horas a 37 ${ }^{\circ} \mathrm{C}$ y finalmente se mide el diámetro del halo formado por la actividad del compuesto. En el ensayo se utilizaron como controles positivos diferentes antibióticos, Ampicilina (50 $\left.\mathrm{mg} \mathrm{mL} \mathrm{m}^{-1}\right)$, Kanamicina $\left(10 \mathrm{mg} \mathrm{mL}^{-1}\right)$ y Tetraciclina $(4,12$ $\mathrm{mg} \mathrm{mL}^{-1}$ ) a una dilución 1:100 en PBS y como controles negativos se empleó DMSO y PBS, de cada control se sirvieron $8 \mu \mathrm{L}$ de cada uno por pozo. El diámetro de las zonas claras o halos de inhibición de crecimiento bacteriano causado por el compuesto en análisis, se midió en mm y los resultados se reportaron como Unidades de Actividad de acuerdo a la relación que establece que 1 Unidad de Actividad (UA) es igual a $0,1 \mathrm{~mm}$ del halo de inhibición.

\section{RESULTADOS Y DISCUSIÓN}

Del extracto de éter de petróleo de hojas de $U$. guianensis fueron aislados e identificados 3 compuestos; una mezcla de dos esteroles, $\beta$-sitosterol y estigmasterol, y un compuesto tipo clorina, éster etílico de feoforbida $a \mathbf{1}$ (Figura 1). Las tres sustancias aisladas se reportan por primera vez para esta especie y el éster etílico de feoforbida $a$ se reporta por primera vez para el género Uncaria. El compuesto $\mathbf{1}$ no ha sido reportado anteriormente en plantas; existen reportes de sustancias similares como la feoforbida $a$, aislada de Berberis fremontii (Stermitz et al. 2000), B. aetnensis (Musumeci et al. 2003) y Artemisia capillaris (Ohshima et al. 1994).

La mezcla de estigmasterol y $\beta$-sitosterol corresponde a un sólido blanco con punto de fusión $138-141^{\circ} \mathrm{C}$. Las estructuras de los componentes de la mezcla fueron elucidadas por comparación de los datos de $\mathrm{RMN}{ }^{1} \mathrm{H}$ obtenidos con los reportados en la literatura para una mezcla de estas sustancias (De Andrade 2003). En el espectro RMN ${ }^{1} \mathrm{H}$ se observa un perfil característico para compuestos esteroidales, con señales intensas en la región de $\delta 0,68$ a $\delta 2,35$ para metilenos y metilos. En $\delta 3,52(1 \mathrm{H}, d d d, J=5,5 \mathrm{~Hz}, 11,9 \mathrm{~Hz}, 17,8$ $\mathrm{Hz})$ se observa una señal caracteristica para el hidrógeno ubicado en la posición 3 del anillo esteroidal, que de acuerdo a las constantes de acoplamiento permiten ubicar el grupo hidroxilo en posicion $\beta$. Las señales en $\delta 5,02(1 \mathrm{H}, d d, J=$ $8,6 \mathrm{~Hz}, 15,2 \mathrm{~Hz})$ y $\delta 5,15(1 \mathrm{H}, d d, J=8,6 \mathrm{~Hz}, 15,2 \mathrm{~Hz})$ son caracteristicas de los protones vinílicos sobre C-22 y C-23 del estigmasterol. Otra señal caracteristica para esteroles es la observada en el espectro en $\delta 5,35(1 \mathrm{H}, d, J=4,9 \mathrm{~Hz})$, correspondiente al protón olefínico en posición 6 del núcleo esteroidal. La mezcla fue analizada por CG, determinándose la siguiente composición: estigmasterol $(17,87 \%)$ y $\beta$-sitosterol
(82,13\%). Los tiempos de retención son 35,5 min y 37,3 min respectivamente.

El compuesto 1 fue aislado como un sólido amorfo de color verde. El compuesto mostró la presencia de un ión pseudomolecular $[\mathrm{M}+\mathrm{H}]^{+}$en $\mathrm{m} / \mathrm{z}$ 621,7431 (calc. 621,7436) en ESI-EMAR, equivalente a la fórmula molecular $\mathrm{C}_{37} \mathrm{H}_{41} \mathrm{~N}_{4} \mathrm{O}_{5}$, presentando así veinte grados de insaturación. En el espectro IR se observan bandas en: $3392 \mathrm{~cm}^{-1}$ (tensión N-H); 1738 y $1698 \mathrm{~cm}^{-1}$ (tensión $\mathrm{C}=\mathrm{O}$ ); $1162 \mathrm{~cm}^{-1}$ (tensión C-O) y 1619 $\mathrm{cm}^{-1}$ (tensión $\mathrm{C}=\mathrm{C}$ ). En el espectro de absorción visible se observan dos bandas $409 \mathrm{~nm}$ (banda Q) y $665 \mathrm{~nm}$ (banda de Soret), que son características de compuestos porfirínicos (Ohshima et al. 1994; Da Silva Soares 2006), lo que permite identificar al compuesto 1 como una porfirina.

El análisis del espectro $\mathrm{RMN}{ }^{1} \mathrm{H}$ permite establecer la presencia de señales para 38 protones. Se observa una seńal en $\delta 3,88(s, 3 \mathrm{H})$ característica de protones de un grupo metoxilo (Silverstein et al. 1991) y 6 señales de protones olefínicos tipo metino en $\delta 6,17(1 \mathrm{H}, d, J=11,5 \mathrm{~Hz}), 6,28(1 \mathrm{H}, d, J=17,8$ $\mathrm{Hz}), 7,98(1 \mathrm{H}, d d, J=11,5 \mathrm{~Hz}, 17,9 \mathrm{~Hz}), 8,56(1 \mathrm{H}, s), 9,36$ $(1 \mathrm{H}, s)$ y $9,50(1 \mathrm{H}, s)$. De estas señales se deduce la presencia del grupo vinilo como sustituyente por el acoplamiento del protón en $\delta 7,98(1 \mathrm{H}, d d, J=11,5 \mathrm{~Hz}, 17,9 \mathrm{~Hz})$ con el protón en $\delta 6,17(2 \mathrm{H}, d, J=11,5 \mathrm{~Hz})$ con una constante de

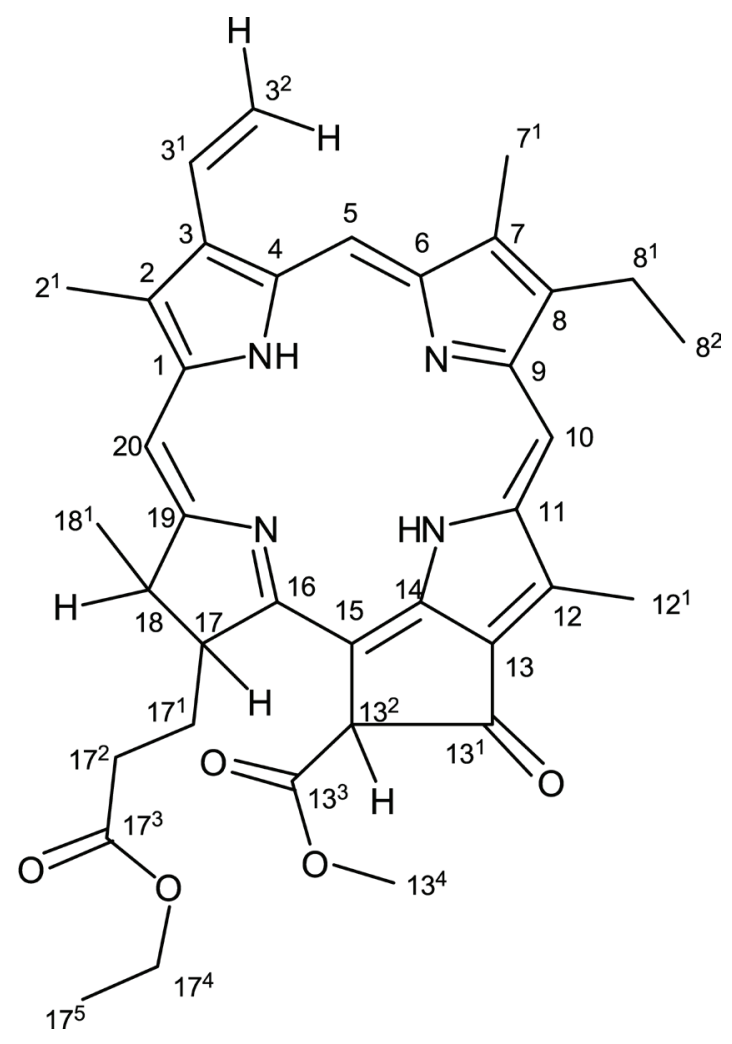

Figura 1 - Estructura del éster etílico de feoforbida a. 
acoplamiento típica para protones olefínicos en cis, y con el protón en $\delta 6,28(2 \mathrm{H}, d, J=17,8 \mathrm{~Hz})$ con una constante de acoplamiento característica de protones olefínicos en trans, lo cual fue corroborado por las correlaciones observadas en el experimento COSY (Figura 2). También se observa la presencia de un fragmento alifático ramificado de 5 miembros, con señales en $\delta 1,81\left(3 \mathrm{H}, d, J=7,2 \mathrm{~Hz} ; \mathrm{H}-18^{1}\right), 4,45(1 \mathrm{H}$, $d q, J=1,6 \mathrm{~Hz}, 7,1 \mathrm{~Hz} ; \mathrm{H}-18), 4,21(1 \mathrm{H}, t d, J=1,7 \mathrm{~Hz}, 7,1$ $\mathrm{Hz} ; \mathrm{H}-17), 2,62\left(2 \mathrm{H}, m ; \mathrm{H}-17^{1}\right)$ y $2,35\left(2 \mathrm{H}, m ; \mathrm{H}-17^{2}\right)$, donde los desplazamientos de las señales indican que dicho fragmento debe estar unido a un grupo desprotector y el cual fue corroborado y asignado por las correlaciones observadas en los experimentos COSY y HMBC, como se observa en la Figura 2. En el espectro RMN ${ }^{1} \mathrm{H}$ también se observan señales para un grupo etilo en $\delta 1,11(3 \mathrm{H}, t, J=7,1 \mathrm{~Hz})$ y $4,02(2 \mathrm{H}$, $q, J=7,2)$; que por sus desplazamientos sugiere que debe estar unido a heteroátomo (Silverstein et al. 1991). Los datos espectroscópicos se resumen en la Tabla 1.

El espectro RMN ${ }^{13} \mathrm{C}$, junto con los experimentos DEPT 90 y DEPT 135 permiten establecer la presencia de señales para 37 átomos de carbono, que incluyen seńales de $7 \mathrm{CH}_{3}$,

Tabla 1 - Datos espectroscópicos de RMN ${ }^{1} \mathrm{H}$ y ${ }^{13} \mathrm{C}$ del compuesto 1.

\begin{tabular}{|c|c|c|c|c|c|c|}
\hline C & ${ }^{13} \mathrm{C}(\mathrm{ppm})$ & ${ }^{1} \mathrm{H}(\mathrm{ppm})$ & MULT. & $\mathrm{J}(\mathrm{Hz})$ & DEPT & $\mathrm{HMBC}$ \\
\hline 1 & 142,0 & - & - & - & C & - \\
\hline 2 & 131,9 & - & - & - & C & - \\
\hline $2^{1}$ & 12,08 & 3,39 & s & - & $\mathrm{CH}_{3}$ & 131,$9 ; 142,0$ \\
\hline 3 & 128,9 & - & - & - & $\mathrm{C}$ & - \\
\hline $3^{1}$ & 129,1 & 7,98 & $\mathrm{dd}$ & 11,$5 ; 17,9$ & $\mathrm{CH}$ & 131,9 \\
\hline $3^{2}$ & 122,8 & $6,17 / 6,28$ & $d / d$ & $11,5 / 17,8$ & $\mathrm{CH}_{2}$ & 128,$9 ; 136,6$ \\
\hline 4 & 136,6 & - & - & - & $\mathrm{C}$ & - \\
\hline 5 & 97,5 & 9,36 & s & - & $\mathrm{CH}$ & 136,$2 ; 136,6$ \\
\hline 6 & 155,7 & - & - & - & C & - \\
\hline 7 & 136,2 & - & - & - & C & - \\
\hline $7^{1}$ & 11,2 & 3,22 & s & & $\mathrm{CH}_{3}$ & 136,$2 ; 145,2 ; 155,7$ \\
\hline 8 & 145,2 & - & - & - & $\mathrm{C}$ & - \\
\hline $8^{1}$ & 19,4 & 3,66 & $t$ & 7,4 & $\mathrm{CH}_{2}$ & 17,$4 ; 136,2 ; 145,2 ; 151,0$ \\
\hline $8^{2}$ & 17,4 & 1,69 & $\mathrm{t}$ & 7,6 & $\mathrm{CH}_{3}$ & 19,$4 ; 145,2$ \\
\hline 9 & 151,0 & - & - & - & $\mathrm{C}$ & - \\
\hline 10 & 104,4 & 9,50 & s & - & $\mathrm{CH}$ & 129,$1 ; 137,9 ; 145,2$ \\
\hline 11 & 136,2 & - & - & - & C & - \\
\hline 12 & 137,9 & - & - & - & C & - \\
\hline $12^{1}$ & 12,1 & 3,69 & s & - & $\mathrm{CH}_{3}$ & 129,$1 ; 136,2 ; 137,9$ \\
\hline 13 & 129,1 & - & - & - & $\mathrm{C}$ & - \\
\hline $13^{1}$ & 189,7 & - & - & - & C & - \\
\hline $13^{2}$ & 64,7 & 6,26 & s & - & $\mathrm{CH}$ & 105,$2 ; 149,7 ; 169,5 ; 189,7$ \\
\hline $13^{3}$ & 169,5 & - & - & - & C & - \\
\hline $13^{4}$ & 52,8 & 3,88 & s & - & $\mathrm{CH}_{3}$ & 169,5 \\
\hline 14 & 149,7 & - & - & - & $\mathrm{C}$ & - \\
\hline 15 & 105,2 & - & - & - & C & - \\
\hline 16 & 161,2 & - & - & - & C & - \\
\hline 17 & 51,1 & 4,21 & $\mathrm{td}$ & 1,$7 ; 8,2$ & $\mathrm{CH}$ & 23,$0 ; 29,9 ; 31,2$ \\
\hline $17^{1}$ & 29,9 & 2,62 & $\mathrm{~m}$ & - & $\mathrm{CH}_{2}$ & 172,9 \\
\hline $17^{2}$ & 31,2 & 2,35 & $\mathrm{~m}$ & - & $\mathrm{CH}_{2}$ & 172,9 \\
\hline $17^{3}$ & 172,9 & - & - & - & $\mathrm{C}$ & - \\
\hline $17^{4}$ & 60,5 & 4,02 & $q$ & 7,2 & $\mathrm{CH}_{2}$ & 172,9 \\
\hline $17^{5}$ & 14,1 & 1,11 & $t$ & 7,1 & $\mathrm{CH}_{3}$ & 60,5 \\
\hline 18 & 50,1 & 4,45 & $\mathrm{dq}$ & 1,$6 ; 7,1$ & $\mathrm{CH}$ & $\begin{array}{c}23,0 ; 29,9 ; 51,1 ; 105,2 ; 161,2 ; \\
172,2\end{array}$ \\
\hline $18^{1}$ & 23,0 & 1,81 & $d$ & 7,2 & $\mathrm{CH}_{3}$ & 50,$1 ; 51,1 ; 172,2$ \\
\hline 19 & 172,2 & - & - & - & $\mathrm{C}$ & - \\
\hline 20 & 93,1 & 8,56 & s & - & $\mathrm{CH}$ & 50,$1 ; 131,9 ; 142,0$ \\
\hline
\end{tabular}


$5 \mathrm{CH}_{2}, 7 \mathrm{CH}$ y 18 carbonos cuaternarios; donde 3 de estos de acuerdo al desplazamiento químico, corresponden a carbonos de grupo carbonilo, dos de grupo éster $(\delta 169,5$ y 172,9$)$ y uno de cetona $(\delta 189,7)$ (Silverstein et al. 1991).

Las correlaciones observadas en el experimento HMBC (Tabla 1 y Figura 2) permiten confirmar los fragmentos propuestos anteriormente. Específicamente la correlación de los protones $\mathrm{H}-17^{1}$ que aparecen en $\delta 2,62$ (pertenecientes al fragmento alifático ramificado de 5 miembros) con el carbono en $\delta 172,9\left(\mathrm{C}-17^{3}\right)$, permite establecer que el fragmento alifático esta unido a un grupo carbonilo de éster. Además, la correlación entre el protón en $\delta 6,26\left(\mathrm{H}-13^{2}\right)$ y los carbonos en $\delta 105,2(\mathrm{C}-15), \delta 149,7(\mathrm{C}-14), \delta 169,6\left(\mathrm{C}-13^{3}\right)$ y $\delta$ $189,6\left(\mathrm{C}-13^{1}\right)$, y la correlación entre el protón en $\delta 3,88(\mathrm{H}-$ $\left.13^{4}\right)$ con el carbono en $\delta 169,6\left(\mathrm{C}-13^{3}\right)$, permiten proponer como sustituyente un $\beta$-cetoéster metílico con sustituyente vinílico en la posición $\alpha$. La presencia del éster etílico puede corroborarse por la correlación de los protones en $\delta 4,02(\mathrm{H}$ $\left.17^{4}\right)$ con el carbono carbonílico en $\delta 172,9\left(\mathrm{C}-17^{3}\right)$.

Al comparar los datos espectroscópicos obtenidos con los reportados en la literatura para compuestos porfirínicos, específicamente los de tipo clorina (Da Silva Soares 2006; Taniguchi et al. 2007), se encontró que los desplazamientos encontrados para el núcleo básico coinciden con los reportados para la feoforbida $a$ (Ohshima et al. 1994), la única diferencia encontrada es la presencia de las señales del grupo etilo perteneciente al éster etílico.

Por todo lo anterior, se concluye que el compuesto 1 aislado de las hojas de U. guianensis corresponde a un compuesto tipo clorina denominado éster etílico de feoforbida a, sustancia que hasta el momento no ha sido aislada de alguna otra especie vegetal. Se reportan por primera vez los datos espectróscopicos para el éster etílico de feoforbida $a$, ya que no aparecen reportados en la literatura.

\section{Resultados de la evaluación de la actividad antibacteriana}

La Tabla 2 resume los resultados obtenidos en la evaluación in vitro de la actividad antibacteriana del éster etílico de feoforbida $a$ frente dos cepas Gram (+): S. aureus ATCC 6538 y E. faecalis ATCC 29212 y contra tres cepas Gram (-): E. coli ATCC 25922, S. typhimurium ATCC 14028s y $S$. typhimurium MS7953.

Los resultados encontrados para el éster etílico de feoforbifda a manifiestan que 4 de las 5 especies microbianas estudiadas son sensibles ante la presencia de este compuesto, siendo S. aureus ATCC 6538 la cepa más sensible. Lo anterior indica claramente que la porfirina evaluada no exhibe selectividad entre bacterias Gram (-) y Gram (+). El compuesto 1 exhibe una actividad antibacteriana menor que la presentada
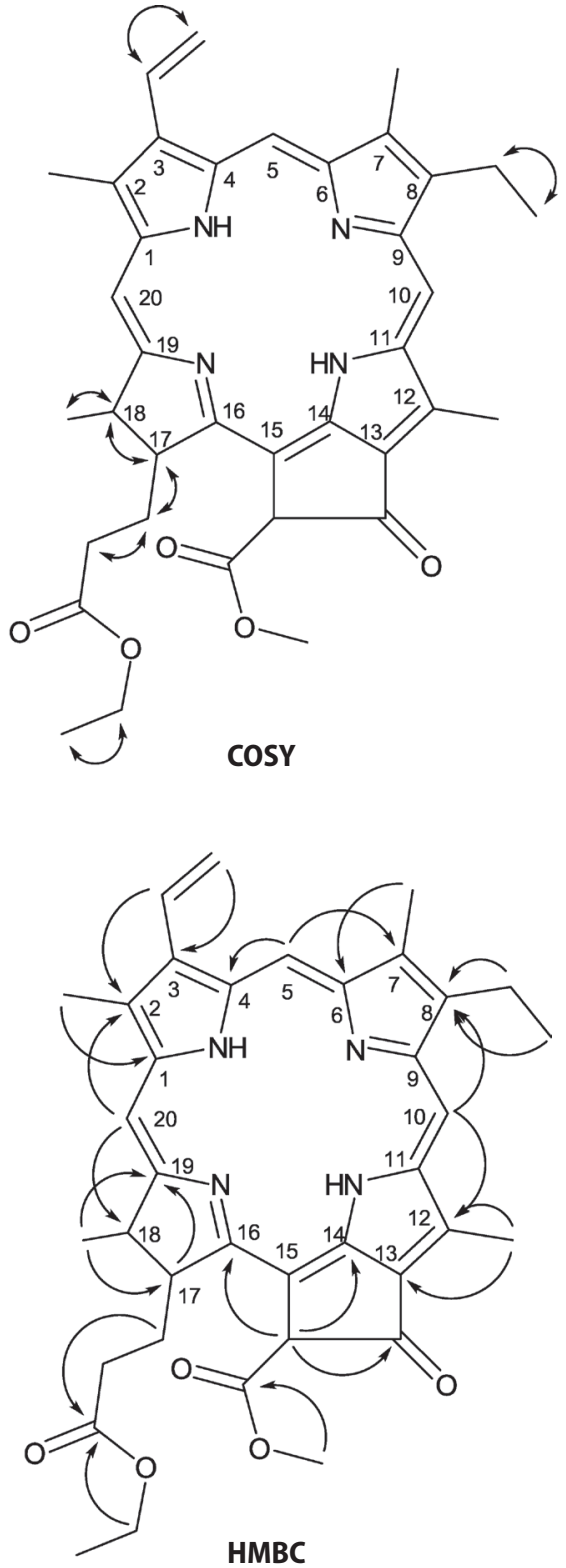

Figura 2 - Correlaciones COSY y HMBC para el compuesto 1.

por los antibióticos de control a la dosis evaluada, sin embargo, es un resultado interesante no reportado anteriormente, que contribuye a la caracterización en cuanto a las propiedades biológicas del compuesto 1 y que sugieren la realización de ensayos más específicos para 1 y compuestos similares en búsqueda de antibacterianos con óptima relación actividad/ toxicidad. Es importante resaltar que el éster etílico de feoforbida $a$ es un compuesto no hemolítico. 
Tabla 2 - Resultados de actividad antibacteriana en el ensayo de difusión radial.

\begin{tabular}{llc}
\hline Cepas inhibidas & Sustancia Evaluada & UA \\
\hline \multirow{4}{*}{ E. faecalis 29212 } & Ampicilina & 120 \\
& Kanamicina & 90 \\
& Tetraciclina & 90 \\
& Compuesto $1(2 \mu \mathrm{g} / \mu \mathrm{L})$ & 50 \\
\hline \multirow{5}{*}{ S. aureus 6538 } & Ampicilina & 110 \\
& Kanamicina & 60 \\
& Tetraciclina & 90 \\
\hline \multirow{5}{*}{ S. typhimurium 14028s } & Compuesto $1(2 \mu \mathrm{g} / \mu \mathrm{L})$ & 50 \\
& Ampicilina & 170 \\
& Kanamicina & 80 \\
& Tetraciclina & 110 \\
& Compuesto $1(2 \mu \mathrm{g} / \mu \mathrm{L})$ & 40 \\
\hline \multirow{5}{*}{ S. typhimurium MS7953 } & Ampicilina & 140 \\
& Kanamicina & 90 \\
& Compuesto $1(2 \mu \mathrm{g} / \mu \mathrm{L})$ & 50 \\
\hline \multirow{5}{*}{ E. coli 25922 } & Ampicilina & 70 \\
& Kanamicina & 90 \\
\cline { 2 - 3 } & Tetraciclina & 100 \\
& Compuesto $1(2 \mu \mathrm{g} / \mu \mathrm{L})$ & 40 \\
\hline
\end{tabular}

aDe cada sustancia se adicionaron $8 \mu \mathrm{L}$

bUnidades de actividad.

\section{AGRADECIMIENTOS}

Al grupo de Productos Naturales Vegetales y a la Universidad Nacional de Colombia por la financiación del trabajo. También agradecemos al FIDIC (Fundación Instituto de Inmunología de Colombia) por la evaluación de la actividad antibacteriana y al Laboratorio de Resonancia Magnética Nuclear de la Universidad Nacional de Colombia por la toma de los espectros de RMN.

\section{BIBLIOGRAFIA CITADA}

Álvarez, C.; Sánchez, O.; Stilke, R.; Lock de Ugaz, O. 1988. Algunos constituyentes de Uncaria guianensis. Revista de Quimica, 2: 99-104.

Aquino, R.; Simone, F.; Pizza, P.; Conti, C.; Stein, L.M. 1989. Plant metabolites: structure and in vitro antiviral activity of quinovic acid glycosides from Uncaria tomentosa and Guettarda platypoda. Journal of Natural Products, 52: 679-685.

Aquino, R.; de Feo, V.; Simone, F.; Pizza, C.; Cirino, G. 1991. Plant metabolites. New compounds and anti-inflamatory activity of Uncaria tomentosa. Journal of Natural Products, 54: 453-459.

Carbonezi, C.A.; Hamerski, L.; Flausino Jr., O.; Furlan, M.; Da Silva, V.; Marx, M.C. 2004. Determinação por RMN das configuraçôes relativas e conformaçóes de alcalóides oxindólicos isolados de Uncaria guianensis. Quimica Nova, 27: 878-881

Da Silva Soares, R.R. 2006. Estudo de propriedades da clorofila a e da feofitina a visando a terapia fotodinâmica. Tesis de Maestría en Química, Universidade Estadual de Mirangá, Mirangá. 29-31 pp.
De Andrade, M.R. 2003. Alcalóides de rutaceae: Química e actividade biológica. Tesis de Doctorado, Universidade Federal de São Carlos, Brasil. 79 pp.

Dreifuss, A.A.; Pereira, A.L.; Avila, T.V.; Da Silva, B.; Rivero, A.J.; Aguilar, J.L.; Acco, A. 2010. Antitumoral and antioxidant effects of a hydroalcoholic extract of cat's claw (Uncaria tomentosa) (Willd. Ex Roem. \& Schult) in an in vivo carcinosarcoma model. Journal of Ethnopharmacology, 130: 127-133.

Gonçalves, C.; Dinis, T.; Batista, M. 2005. Antioxidant properties of proanthocyanidins of Uncaria tomentosa bark decoction: a mechanism for anti-inflammatory activity. Phytochemistry, 66: 89-98

Heitzman, M.; Neto, C.; Winiarz, E.; Vaisberg, A.; Hammond, G. 2005. Ethnobotany, phytochemistry and pharmacology of Uncaria (Rubiaceae). Phytochemistry, 66: 5-29.

Huang, K.C. 1999. The pharmacology of Chinese herbs. Hardcover Book Press Co. Florida. 125pp.

Keplinger, K.; Laus, G.; Wurm, M.; Dierich, M.; Teppner, H. 1999. Uncaria tomentosa (Willd.) DC. Ethnomedicinal use and new pharmacological, toxicological and botanical results. Journal of Ethnopharmacology, 64: 23-34.

Laus, G.; Keplinger, K. 2003. Alkaloids of peruvian Uncaria guianensis (Rubiaceae). Phyton, 43: 1-8.

Laus, G.; Keplinger, K.; Brossner, D. 1997. Alkaloids of peruvian Uncaria tomentosa. Phytochemistry, 45: 855-860.

Lavault, M.; Moretti, C.; Bruneton, J. 1983. Alcaloïdes de I'Uncaria guianensis. Journal of Medicinal Plant Research, 47: 244-245.

Lehrer, R.; Rosenman, M.; Harwig, S.; Jackson, R.; Eisenhauer, P. 1991. Ultrasensitive assay for endogenous antimicrobial polypeptides. Journal of Inmunological Methods, 137: 167-173.

Mendoza, H.; Ramírez, B.; Jiménez, L. 2004. Rubiaceae de Colombia. Guía Ilustrada de Géneros. Instituto de Investigación de Recursos Biológicos Alexander von Humboldt. 128 pp.

Muhammad, I.; ChuckDunbar, D.; Khan, R.; Ganzera, M.; Khan, I. 2001. Investigation of Uńa de Gato I. 7-Deoxyloganic acid and ${ }^{15} \mathrm{~N}$ NMR sprectroscopic studies on pentacyclic oxindole alkaloids from Uncaria tomentosa. Phytochemistry, 57: 781-785.

Musumeci, R.; Speciale, A.; Costanzo, R.; Annino, A.; Ragusa, S.; Rapisarda, A.; Pappalardo, M.S.; Iauk, L. 2003. Berberis aetnensis C. Presl. extracts: antimicrobial properties and interaction with ciprofloxacin. International Journal of Antimicrobial Agents, 22: 48-53.

Ohshima, T.; Hirata, M.; Oda, T.; Sasaki, A.; Shiratsuchi, M. 1994. Pheophorbide $a$, a potent endothelin receptor antagonist for both $\mathrm{ET}_{\mathrm{A}}$ and $\mathrm{ET}_{\mathrm{R}}$ subtypes. Chemical \& Pharmaceutical Bulletin, 42: 2174-2176.

Pengsuparp, T.; Indra, B.; Nakagawasai, O.; Tadano, T.; Mimaki, Y.; Sashida, Y.; Ohizumi, Y.; Kisara, K. 2001. Pharmacological studies of geissoschizine methyl ether, isolated from Uncaria sinensis Oliv. in the central nervous system. European Journal of Pharmaceutical Sciences, 425: 211-218.

Pereira, R.; Valente, L.; Pinto, J.; Bertolucci, S.; Bezerra, G.; Alves, F.; Dos Santos, P.; Benevides, P.; Siani, A.; Rosario, S.; Mazzei, J.; Avila, L.; Gomes, L.; Aquino-Neto, F. 2008. In vitro cultivated 
Uncaria tomentosa and Uncaria guianensis with determination of the pentacyclic oxindole alkaloid contents and profiles. Journal of the Brazilian Chemical Society, 19: 1193-1200.

Pilarski, R.; Zieli'nski, H.; Ciesiołka, D.; Gulewicz, K. 2006. Antioxidant activity of ethanolic and aqueous extracts of Uncaria tomentosa (Willd.) DC. Journal of Ethnopharmacology, 104: 18-23.

Rojas, S. 2001. Especies promisorias de la Amazonía. Conservación, manejo y utilización del germoplasma. Corporación Colombiana de Investigación Agropecuaria - Corpoica y Conciencias. CI Macagual, Caquetá, Putumayo.1: 183-192.

Senatore, A.; Cataldo, A.; Iaccarino, F.P.; Elberti, M.G. 1989. Ricerche fitochimiche e biologiche sull' Uncaria timentosa. Bolletino della Societa Italiana di Biologia Sperimentale, 65: 517-520.

Shim, J.S.; Kim, H.G.; Ju, M.S.; Choi, J.G.; Jeong, S.Y.; Oh, M.S. 2009. Effects of the hook of Uncaria rhynchophylla on neurotoxicity in the 6-hydroxydopamine model of Parkinson's disease. Journal of Ethnopharmacology, 126: 361-365.

Silverstein, R.M.; Bassler, G.C.; Morril, T.C. 1991. Spectrometric Identification of Organic Compounds. Fifth Edition. London: John Wiley \& Sons. p. 95-129.

Smith, K.M.; Goff, D.A; Abraham, R.J. 1987. NMR spectra of porphyrins. 29. conformation of the propionic ester side chain in chlorophyll derivatives. Journal of Organic Chemistry, 52: 176-180

Spangler, D.; Maggiora, J.; Shipman, L.; Christoffersen, R. 1977. Stereoelectronic properties of photosynthetic and related systems. 1. Ab iInitio quantum mechanical ground state characterization of free base porphine, chlorin, and ethyl pheophorbide a. Journal of the American Chemical Society, 99: 7470-7477.
Stermitz, F.; Tawara-Matsuda, J.; Lorenz, P.; Mueller, P.; Zenewicz, L.; Lewis, K. 2000. 5'-methoxyhydrocarpin-D and pheophorbide A: Berberis species components that potentiate berberine growth inhibition of resistant Staphylococcus aureus. Journal of Natural Products, 63: 1146-1149.

Taniguchi, M.; Ptaszek, M.; McDowell, B.; Boyle, P.; Lindsey, J. 2007. Sparsely substituted chlorins as core constructs in chlorophyll analogue chemistry. Part 3: Spectral and structural properties. Tetrahedron, 63: 3850-3863.

Trejo, G.; Cerda, C.; Rodríguez, M.; Ramos, A. 2005. Monoterpenoid oxindol alkaloid production by Uncaria tomentosa (willd.) D.C. Cell suspension cultures in stirred tank bioreactor. Biotechnology Progress, 21: 786-792.

Valente, L.; Bizarri, C.; Liechocki, S.; Barboza, R.; Paixão, D.; Almeida, M. B.; Benevides, P.J.; Magalhães, A.; Siani, A.B. 2009. Kaempferitrin from Uncaria guianensis (Rubiaceae) and its potential as a chemical marker for the species. Journal of the Brazilian Chemical Society, 20: 1041-1045.

Wang, Z. 1989. Quantitative determination of the alkaloids of $U$. rhynchophylla by calcium chromatography. ChineseTraditional and Herbal Drugs, 20:11-20.

Yépez, A.M.; Lock de Ugaz, O.;. Alvarez, C.M.; De Feo, V.; Aquino, R.; De Simone, F.; Pizza, C. 1991. Quinovic acid glycosides from Uncaria guianensis. Phytochemistry, 30: 1635-1637.

Zeballos, P.A.; Tomazello, M. 2006. Anatomia do lenho de Uncaria guianensis e U. tomentosa (Rubiaceae) do estado do Acre, Brasil. Acta Amazonica, 36: 169-176.

Recebido em 9/03/2010

Aceito em $7 / 07 / 2010$ 